\title{
Research Progress on the Neuroprotective Effect of Leonurine on Ischemic Stroke
}

\author{
Honglong Wu $\mathrm{W}^{1,2}$, Long He ${ }^{1,2}$, Zhifen Tan1, Meikui Zhang1,2* \\ 1Shaanxi University of Chinese Medicine, Xianyang 712046, Shaanxi Province, China \\ ${ }^{2}$ The General Hospital of the People's Liberation Army, Beijing 100853, China \\ *Corresponding author: Meikui Zhang, zmk301@126.com
}

\begin{abstract}
Leonurus is one of the commonly used clinical drugs in traditional Chinese medicine. In recent years, leonurus has been widely used for cerebrovascular diseases. With the deepening of research, the effect of its main component, leonurine, on the central nervous system has become clearer, and its mechanism has been confirmed and explained from many aspects. For reference, this paper discusses the latest research on the neuroprotective effect of leonurine on ischemic stroke at home and abroad in recent years.
\end{abstract}

Keywords: Leonurine; Ischemic stroke; Neuroprotection

Publication date: November 2021; Online publication: November 30, 2021

\section{Introduction}

Stroke is a common type of cerebrovascular disease, of which ischemic stroke accounts for about $70 \%$ of all strokes ${ }^{[1]}$. At present, reperfusion in a specific time window is the standard for the treatment of ischemic stroke, but the injury after ischemia-reperfusion (I/R) is inevitable; this includes oxidative stress, inflammatory response, and so on ${ }^{[2]}$.

Modern pharmacological studies have proved that one of the effective components of leonurus is leonurine. Its effects include reducing oxidative stress and apoptosis, inhibiting inflammatory factors, preventing platelet aggregation, and so on ${ }^{[3]}$. Leonurine has high therapeutic value for a variety of diseases, especially for cardiovascular and cerebrovascular diseases. It is considered as a potential drug for the treatment of cardiovascular and central nervous system diseases ${ }^{[4]}$.

\section{Alleviate oxidative stress after stroke}

After ischemic stroke, cerebral ischemia, hypoxia, and I/R lead to excessive production of reactive oxygen species (ROS), resulting in oxidative stress and damage of lipids, proteins, and DNA in cells. When Shuai Du and other researchers studied the pathological changes of brain tissues induced by leonurine in rats with ischemic stroke based on PI3K / Akt / NF- $\kappa \mathrm{B}$ signaling pathway, they found that the neurological impairment score, nitric oxide (NO), endothelial nitric oxide synthase (NOS) content, and the expression

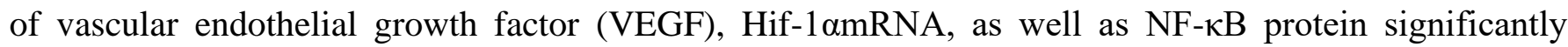
decreased, while ox-LDL, superoxide dimutase (SOD), and the expression of AKT as well as PI3K protein significantly increased in rats that received leonurine ${ }^{[5]}$. It has been suggested that leonurine can reduce nerve injury and oxidative stress response after infarction. In another study ${ }^{[6]}$, rats with middle cerebral artery occlusion (MCAO) were treated with leonurine. The study found that leonurine can increase the expression of acetylase HDAC4 and inhibit the expression of NADPH oxidase (NOX) as well as matrix 
metalloproteinase 9 (MMP-9).

\section{Reduce apoptosis after stroke}

Qi Jia and other researchers proposed that leonurine can significantly reduce apoptosis after ischemic stroke; its mechanism may be through NF- $\kappa \mathrm{B}$ and the activation of mitogen-activated protein kinase (MAPK) in massive cells to significantly reduce the expression of Bax, increase Bcl-2, and increase the content of cytochrome $\mathrm{C}$ (cyt-C) isolated from the ischemic cortex in mitochondria, so as to play an anti-apoptotic role $^{[7]}$. A few other researchers treated transient middle cerebral artery occlusion in rats with leonurine and found that leonurine reduces cell degeneration and apoptosis ${ }^{[8]}$; leonurine can prevent oxygen glucose deprivation/re-oxygenation (OGD/R)-induced apoptosis, increase cell viability, decrease the number of TUNEL positive cells, accompanied by the upregulation of $\mathrm{Bcl}-2$ and $\mathrm{Bcl}-\mathrm{xL}$ proteins with the downregulation of Bax protein. It has been suggested that leonurine delays neuronal damage and cell apoptosis through the p-STAT3/NOX4/Bcl-2 signaling pathway.

\section{Inhibit post-stroke inflammatory factors}

After ischemic stroke, over-activated inflammatory response can result in secondary neuronal injury. In a study, Zhiliang $\mathrm{Hu}$ and other researchers pretreated cells with leonurine and then induced them with hypoxia/reoxygenation to test their viability. The results showed that the survival rate of cells pretreated with leonurine increased, the secretion volume of TNF- $\alpha$, IL-1 $\beta$, and IL-6 decreased, the protein

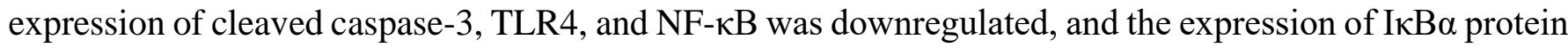
was upregulated ${ }^{[9]}$, suggesting that leonurine can inhibit inflammation by inhibiting the TLR4/NF- $\mathrm{B}$ signaling pathway. The results from treating neonatal ischemic and hypoxic rats with leonurine showed that the symptoms and levels of TNF- $\alpha$ and IL- $1 \beta$ of the rats in the leonurine-treated group significantly reduced compared with those of the control group. The results suggest that leonurine can reduce the symptoms of hypoxic-ischemic brain injury (HIBI) in neonatal rats by reducing the inflammatory response of brain tissue [10].

\section{Prevent platelet aggregation after stroke}

During cerebral ischemia or hypoxia and I/R, a large number of platelets adhere to the vascular wall of the lesion, increasing the risk of secondary thrombosis and brain tissue injury. In a study, Juan Liu and other researchers took blood from the femoral artery of Sprague-Dawley (SD) rats, mixed it with citric acid, and centrifuged to obtain a supernatant; leonurine was then separated from leonurus sibiricus, and then the rate of platelet aggregation induced by adenosine diphosphate (ADP) was measured by a platelet aggregation instrument based on Born's turbidimetric method; the study found that leonurine has a significant inhibitory effect on ADP-induced platelet aggregation ${ }^{[11]}$. Another study found that leonurine has a significant effect in reducing blood viscosity, and its guanidine structure can resist platelet aggregation ${ }^{[12]}$.

\section{Discussion}

This paper summarized several experimental studies on leonurine at home and abroad. It has been found that leonurine can improve the neurological deficits and promote nerve regeneration in patients with ischemic stroke by alleviating oxidative stress, reducing apoptosis, inhibiting inflammatory response, and preventing platelet aggregation, thus improving the symptoms and prognosis of patients. It is believed that there would be more clinical research and basic research in the future to enrich the list of drugs for the treatment of ischemic stroke and to provide practical evidence for expanding the application scope of leonurine and its preparations. 


\section{Funding}

This work was supported by the General Project of Beijing Natural Science Funds - Study on the Effect of Naoshuning, a Traditional Chinese Medicine, for Activating Blood, Promoting Blood Circulation, Removing Water, and Dredging the Internal Organs, on Nerve Regeneration and Its Mechanism in Acute Ischemic Stroke (Fund Number: 7182156).

\section{Disclosure statement}

The authors declare that there is no conflict of interest.

\section{References}

[1] Peng B, Wu B, 2018, Guidelines for the Diagnosis and Treatment of Acute Ischemic Stroke in China 2018. Chinese Journal of Neurology, 51(09): 666-682.

[2] $\mathrm{Hu}$ L, Fang R, Guo M, 2020, Knockdown of lncRNA SNHG1 Alleviates Oxygen-Glucose Deprivation/Reperfusion-Induced Cell Death by Serving as a ceRNA for Mir-424 in SH-SY5Y Cells. A Journal of Progress in Neurosurgery, Neurology and Neurosciences, 42(1): 47-54.

[3] Li Y-Y, Lin Y-K, Liu X-H, et al., 2020, Leonurine: From Gynecologic Medicine to Pleiotropic Agent. Chinese Journal of Integrative Medicine, 26(2): 152-160.

[4] Huang L, Xu D-Q, Chen Y-Y, et al., 2021, Leonurine, a Potential Drug for the Treatment of Cardiovascular System and Central Nervous System Diseases. Brain and Behavior, 11(2): e01995.

[5] Du S, Liu J, Liu T, et al., 2019, Discussion on Effects of Leonurine on Brain Histopathological Changes in Rats with Ischemic Stroke Based on PI3K/AKT/NF-kB Signaling Pathway. Chinese Journal of Arteriosclerosis, 27(10): 853-861.

[6] Zhang Q-Y, Wang Z-J, Sun D-M, et al., 2017, Novel Therapeutic Effects of Leonurine on Ischemic Stroke: New Mechanisms of BBB Integrity. Oxidative Medicine and Cellular Longevity, 2017.

[7] Jia Q, Zhen YH, Hong X, et al., 2010, Neuroprotective Effects of Leonurine on Ischemia/ReperfusionInduced Mitochondrial Dysfunctions in Rat Cerebral Cortex. Biological and Pharmaceutical Bulletin, 33(12): 1958-1964.

[8] Zhang Q-Y, Wang Z-J, Miao L, et al., 2019, Neuroprotective Effect of SCM-198 through Stabilizing Endothelial Cell Function. Oxidative Medicine and Cellular Longevity, 2019.

[9] Hu ZL, Chi H, Ding SY, 2021, Effects of Leonurine Pretreatment on Viability and Protein Expression of TLR4/NF- $\kappa$ B Pathway in Hypoxic/Reoxygenated Rat Cardiomyocytes. Journal of Zhengzhou University (Medical Science), 56(04): 521-525.

[10] Du N, 2021, Protective Effect and Mechanism of Leonurine on Hypoxic-ischemic Brain Injury in Neonatal Rats, Dali University, 42.

[11] Liu J, Zhou Q, Peng Q, et al., 2015, Study on Chemical Constituents and Antiplatelet Aggregation Activity of Leonurus Sibiricus. Chinese Traditional Patent Medicine, 37(11): 2439-2442.

[12] Zhang X, 2015, Effects of Aromatic Compounds of Leonurus Herb on Coagulation Function in Vitro in Rats. Chinese Traditional Patent Medicine, 37(07): 1573-1575. 